\title{
Auto-censure et dé-censure dans Nègre de personne de Roland Brival et The Pain Tree d'Olive Senior
}

Self-Censorship and De-Censorship in Roland Brival's Nègre de personne and Olive Senior's The Pain Tree

\section{Patricia Donatien}

\section{OpenEdition}

Journals

Édition électronique

URL : http://journals.openedition.org/transtexts/1402

DOI : $10.4000 /$ transtexts. 1402

ISSN : 2105-2549

Éditeur

Gregory B. Lee

Référence électronique

Patricia Donatien, «Auto-censure et dé-censure dans Nègre de personne de Roland Brival et The Pain Tree d'Olive Senior », Transtext(e)s Transcultures 跨文本跨文化 [En ligne], 15 | 2020, mis en ligne le 14 décembre 2020, consulté le 26 janvier 2021. URL : http://journals.openedition.org/transtexts/1402 ; DOI : https://doi.org/10.4000/transtexts.1402

Ce document a été généré automatiquement le 26 janvier 2021.

(c) Tous droits réservés 


\title{
Auto-censure et dé-censure dans Nègre de personne de Roland Brival et The Pain Tree d'Olive Senior
}

\author{
Self-Censorship and De-Censorship in Roland Brival's Nègre de personne and \\ olive Senior's The Pain Tree
}

Patricia Donatien

1 Roland Brival et Olive Senior sont deux auteurs caribéens des sphères française et anglophone aux écritures singulières et différentes. Le Martiniquais Roland Brival affectionne les biographies historiques mettant en scène de grands hommes, alors que dans ses recueils, Olive Senior, Jamaïcaine de naissance, substitue aux épopées «brivaliennes" de petits récits et des nouvelles intimistes. Cependant, les deux écrivains ont en commun une passion pour les récits de vie et l'exploration des intimités. Les cheminements de leurs héros ont pour cadre une région caribéenne marquée au fer rouge par les systèmes plantationnaires et coloniaux du début du XX siècle. Par ailleurs, la construction des existences de ces personnages charismatiques est également fondée sur l'interdit: l'interdit de livrer l'émotion, l'interdit d'être soi, l'interdit de dire son expérience, l'interdit de crier l'injustice et enfin, l'interdit d'exprimer sa souffrance. Dès lors, comment parler de soi, comment explorer le «je » et le «moi » profond au milieu de tant d'injonctions au silence; et comment, dans cet environnement coercitif de la société de plantation, réussir à braver la censure et à ne pas s'autocensurer?

2 Roland Brival et Olive Senior, en tant qu'auteurs contemporains issus de ces sociétés coloniales infantilisantes et répressives qui, pendant des siècles, ont imposé le mutisme, pratiquent par leur écriture ce que je nomme la dé-censure. En effet, dans la Guyane et la Jamaïque du début du $\mathrm{XX}^{\mathrm{e}}$ siècle, comme dans les nombreux pays de l'Afrique colonisée :

[1]a censure constitue un instrument de contrôle social à l'usage des autorités. Censurer revient à s'arroger le droit de choisir pour autrui, à s'ériger en instance morale au nom d'un savoir supérieur ou d'un système de valeurs. [...] Afin de 
perpétuer le statu quo, il faut empêcher que ne circulent des idées potentiellement subversives auprès d'un public vulnérable, proie facile de la propagande. Cette analyse peut être aisément transposée dans le cadre colonial, le rapport de classe se doublant d'un rapport de domination culturelle et politique. Au contrôle social et au souci de l'ordre public s'ajoute une rhétorique des colonisateurs sur la masse des colonisés - concept générique - dont on met en doute les capacités intellectuelles et, précisément, l'aptitude à lire... ${ }^{1}$

3 Les œuvres de Brival et de Senior opèrent une déconstruction de ce « contrôle social » et vont inverser cette "rhétorique des colonisateurs » et c'est donc en ce sens qu'il s'agit d'une dé-censure; leurs œuvres deviennent dès lors des espaces dans lesquels l'indicible et l'inaudible vont pouvoir se faire entendre, et les idées aussi subversives soient-elles, circuler. Au travers d'un certain nombre de stratégies d'écriture clairement identifiables comme relevant d'une décolonisation de la pensée, les voix réprimées vont se révéler, dé-censurant ainsi le verbe et la langue. La dé-censure peut donc être définie ici comme une neutralisation de la censure, mais aussi comme une forme de rétablissement de la parole oblitérée.

Les romans présentés dans cet article sont tous deux récents et s'inscrivent dans une dynamique d'affirmation d'une esthétique caribéenne affranchie de la commotion coloniale ; il s'agit d'une part de Nègre de personne de Roland Brival, publié en 2016, et d'autre part de The Pain Tree d'Olive Senior, publié en $2015 .^{2}$ La première de ces deux œuvres est très singulière en ce sens qu'elle met en scène une partie de la vie du poète guyanais Léon Gontran Damas sous la forme d'un « récit autobiographique » fictif dans lequel l'auteur, Roland Brival, devient le double de Damas. Tout au long de ce récit poignant et palpitant, le poète, par la voix de Brival, raconte son voyage à New York en 1939, après la publication de son recueil Pigments sorti en 1937. Damas fuit la guerre qui vient d'être déclarée en Europe, mais il fuit aussi la censure qui frappe l'ensemble de son travail et plus particulièrement son fameux ouvrage Pigments, brûlant recueil de poèmes censuré en raison des appels à la conscience critique qu'il contient, ainsi que des exhortations à la rébellion que lance Damas aux jeunes noirs enrôlés, nouveaux esclaves des temps modernes, comme le dénonce le poète guyanais, et que les conflits internes à l'Europe ne concernent que de loin. Ainsi, Brival va donc, dans Nègre de Personne, substituer son écriture et sa voix à celles de Damas pour dé-censurer l'œuvre du poète guyanais et lui permettre de dire tout ce qu'il n'a pas pu dire ou tout ce qu'il lui a été interdit de dire.

5 Le deuxième roman, The Pain Tree, publié en 2015 par Olive Senior, est une évocation de plusieurs récits de vie, tous mis en relation selon deux axes récurrents et incontournables dans chaque évocation : une femme, la mère de l'auteur et un arbre, dont le roman porte d'ailleurs le nom The Pain Tree. Dans cet ouvrage, en utilisant, comme Brival, la première personne, Olive Senior évoque plusieurs protagonistes de son enfance dont les voix et les désirs ont été réprimés, leur accordant ici, pour la première fois, un auditoire essentiel à l'expression de leur mal-être.

6 Ainsi, la censure dans ces deux œuvres (Nègre de personne et The Pain Tree) n'est pas celle que subissent leurs auteurs ; elle est celle qu'ont subie d'une part Léon Gontran Damas et d'autre part les personnages des campagnes jamaïcaines décrits par Olive Senior villageois, femmes et enfants opprimés par le système colonial. Tous sont victimes d'un empêchement, d'une impossibilité de s'exprimer, d'une censure de leur parole ou de leur écriture, due aux restrictions des droits des sujets coloniaux, à la rigidité d'une institution et de sociétés marquées par le non-humanisme qui prévaut pendant 
l'esclavage et la colonisation et qui impose, comme le dit Aimé Césaire, "l'amnésie comme méthode », une amnésie imposée par la violence, comme l'explique le médecinpsychiatre et écrivain martiniquais Frantz Fanon. ${ }^{3}$ Dans l'exploration du fonctionnement du monde colonial qu'il propose dans les Damnés de la terre, Fanon dit en effet ceci :

Dans les régions coloniales [...] le gendarme, le soldat, par leur présence immédiate, leurs interventions directes et fréquentes, maintiennent le contact avec le colonisé et lui conseillent, à coup de crosse ou de napalm, de ne pas bouger. On le voit, l'intermédiaire du pouvoir utilise un langage de pure violence. L'intermédiaire n'allège pas l'oppression, ne voile pas la domination. Il les expose, le manifeste avec la bonne conscience des forces de l'ordre. L'intermédiaire porte la violence dans les maisons, dans les cerveaux du colonisé. ${ }^{4}$

7 Cette violence portée dans les maisons et les cerveaux des colonisés est inouïe, profonde, elle interdit de penser et applique la violence symbolique qui agentise le colonisé lui-même et le persuade du bienfondé de cette brutalité et du dénigrement dont il est l'objet. Ainsi, la censure participe de la gestion, par l'étouffement, de la pensée et de toute idéologie générant de la résistance ; cette censure prévient autant qu'elle réprime toute production échappant à la subalternité attendue. C'est pour cette raison, et pour expliquer ce phénomène, que Fanon va développer un concept qui demeure peu connu, celui de "l'imposition culturelle», qui nous paraît crucial pour notre propos. En effet, dans le chapitre intitulé "Le nègre et la psychopathologie ", Fanon explique que le comportement et la pensée de celui qu'il nomme «l'Antillais » nous dirions aujourd'hui le Caribéen- sont façonnés par un inconscient collectif, conséquence, [dit-il],

de ce que j'appellerai l'imposition culturelle irréfléchie. Rien d'étonnant, donc, à ce qu'un Antillais, soumis à la méthode du rêve éveillé, revive les mêmes fantasmes qu'un Européen... [I]l est normal que l'Antillais soit négrophobe.... Par l'inconscient collectif le nègre a fait sien tous les archétypes de l'Européen....Sans faire appel à la notion de catharsis collective, il me serait facile de montrer que le nègre irréflexivement, se choisit objet susceptible de porter le péché originel. Pour ce rôle, le blanc choisit le Noir, et le Noir qui est un Blanc choisit le Noir. ${ }^{5}$

Cette imposition culturelle est pernicieuse et pénétrante ; elle s'installe au plus profond des âmes des colonisés grâce à, et par, l'action de la violence symbolique qui persuade de la subalternité et pose les interdits. L'interdit d'être soi débouche donc dans ce contexte sur une autocensure, forme de répression de soi-même, qui frappe puissamment les personnages d'olive Senior et, en partie, le jeune guerrier anticolonialiste Damas, qui se bat contre le colonisé subalterne qui est en lui.

9 Cette étude des textes de Brival et Senior s'articule donc en deux temps : une première partie assez succincte démontre comment les sujets caribéens que campent les personnages de Brival et Senior sont l'objet d'une censure qui revêt trois aspects : la censure de la langue, la censure de l'affect, la censure poétique. Une deuxième partie, qui est l'axe principal de ce travail puisqu'elle prend appui sur les deux romans qui sont au cœur de cette étude, permet de démontrer comment les œuvres contemporaines de Roland Brival et d'olive Senior participent d'une dé-censure contemporaine des voix et de l'écriture caribéennes de la première moitié du XXe siècle. 


\section{Le temps colonial de l'écriture caribéenne : censure, autocensure et transgression}

Je viendrai à ce pays mien et je lui dirais : «Embrassez-moi sans crainte... Et si je ne

sais que parler, c'est pour vous que je parlerai. »

Et je lui dirais encore :

«Ma bouche sera la bouche des malheurs qui n'ont point de bouche, ma voix, la

liberté de celles qui s'affaissent au cachot du désespoir. $»^{6}$

Ce grand cri de la Négritude qui retentit en 1939 est une illustration poétique de la situation dans laquelle se trouve, dans la première moitié $\mathrm{du} \mathrm{XX}^{\mathrm{e}}$ siècle, un grand nombre de peuples prisonniers d'un système qui a cessé officiellement de les catégoriser en objets et qui pourtant continue de pratiquer la "chosification.$^{7}$ Les colonisés que sont les Martiniquais, les Guadeloupéens et les Guyanais avant 1948 (date d'application du décret de 1946 qui accorde la départementalisation aux colonies), comme d'ailleurs tous les sujets britanniques dominés par le régime du Crown Colony System qui régit les colonies, vivent non seulement dans une misère effroyable, mais surtout dans un déni total de leur personne.

11 La censure est donc, pour tout citoyen caribéen de cette époque, un élément du quotidien, elle est une partie intégrante de leur habitus. Leur langue, le créole, est censurée. Seule une minorité des populations des îles françaises et anglaises de l'Arc Caribéen a accès à l'éducation et à la langue officielle du colonisateur; et la masse populaire, qui ne possède aucun autre moyen de communication, subit l'interdiction et le silence. À l'école, dans les administrations, à l'église, dans les tribunaux, seul est autorisé le français ou l'anglais (selon les îles) et toute revendication, explication, ou simple démarche devient dès lors, pour la majorité, impossible.

Pour ce qui est de l'affect, l'esclavage, avant la colonisation, a formaté tous les individus dans l'autocensure. En effet, comment vivre, ou plutôt survivre si l'on se laisse aller à la moindre faiblesse, à la douceur des sentiments, aux larmes, aux plaintes dans un monde où le non-humanisme est loi et où la peur et la violence règnent en maitresses dominatrices? Tout refouler est le moyen de continuer: ne pas parler de ses sentiments, ne pas livrer de ses angoisses, taire les traumatismes subis, serrer les dents et continuer est la seule alternative pour rester en vie.

Les chercheuses jamaïcaines Carole Boyce Davies et Elaine Savory Fido sont les premières à avoir avancé, dans leur ouvrage Out of the Kumbla: Caribbean Women and Literature, paru en 1990, l'idée d'un refoulement total de l'esclavagisé et du colonisé dans une calebasse émotionnelle, la "kumbla», qui lui interdit toute sensibilité et toute expression de soi-une forme d'évitement, de refoulement et d'autocensure salutaire. ${ }^{8}$ Carole Boyce Davis et Elaine Savory Fido vont prôner, dans leur approche, la nécessité, en cette fin de XXe siècle, de briser la calebasse pour sortir de la "kumbla ", et c'est en quelque sorte ce que vont réaliser Roland Brival pour Damas et Olive Senior pour ses compatriotes : leur permettre de parler enfin. On le comprend, l'enfermement des sujets caribéens dans cette calebasse émotionnelle quasi étanche va durer très longtemps et dure sans doute encore, provoquant "la blès ", une blessure sacrée première qui se forme au cœur de l'être qui ne se dit pas, qui refuse de parler de ses douleurs, une blessure qui suppure sans fin. J'explique dans mon ouvrage L'exorcisme de la blès, paru en 2006 , que :

Cette maladie (répertoriée uniquement dans la médecine populaire) serait apparue

[...] dans les populations ayant successivement subi la déportation, l'esclavage et la 
colonisation. Elle découlerait d'un traumatisme fondamental généré par ces régimes déstructurant [...] et par le carcan de souffrance et de déni de soi imposé à chaque individu. La blès serait donc la conséquence de siècles de renoncement et de refoulement imposés à eux-mêmes par les individus prisonniers de ce système.[...] La blès, considérée aujourd'hui comme désuète et relevant de la mythologie présenterait une réelle pertinence quant à l'élucidation d'une certaine difficulté à affronter les souffrances conscientes et à extérioriser les plaies et les lancinements inconscients liés à l'histoire et aux mécanismes de sauvegarde développés à la fois par chaque individu mais aussi par l'ensemble des communautés. ${ }^{9}$

14 Ainsi, la censure poétique est à la fois le résultat de l'imposition culturelle, du musellement colonial mais aussi et surtout d'une autocensure pratiquée par tous ceux qui, impactés par la blès, sont incapables d'extérioriser tous leurs traumas.

Ces trois dimensions de la censure (de la langue, de l'affect et poétique) nous permettent de comprendre comment, dans la structure des récits, le jeu s'établit entre censure, autocensure, et dé-censure, à la fois sur le plan du récit historique, mais aussi dans l'approche sociétale, psychologique et esthétique. Ces trois axes nous autorisent aussi à appréhender les stratégies des auteurs contemporains que sont Brival et Senior et qui réussissent à approcher au plus près ce qui, malgré la trivialité destructrice des antihumanismes déstructurants, constitue l'essentiel de l'être et de son besoin de dire.

\section{La dé-censure : écrire ce qu'ils n'ont pas pu dire pour réparer la parole et édifier l'esthétique caribéenne}

Damas, nous dit l'écrivain guadeloupéen Daniel Maximin dans son article Léon Damas, Étoile pigmentée de graffiti, est un poète qui a commencé par le silence, car prononcer des mots lui a été longtemps difficile. ${ }^{10}$

Il est resté muet jusqu'à l'âge de six ans. Outre-mère et forêts vierges à ses désirs malades. Il est mort le 22 janvier 1978, des suites d'un cancer de la langue. Entre ces deux mutismes s'est élaborée son œuvre : quelques graffiti crayonnés sur les murs de la honte, de l'oppression et de l'indifférence. ${ }^{11}$

17 Et quand la mort s'en est mêlée, l'impossibilité du dire s'est faite encore plus grande. Ainsi, le langage poétique de Damas se forge à partir du silence et de sa langue sans mots. La poésie, en parole essentielle, où les mots se comptent et s'harmonisent, deviendra son refuge, sa méthode et sa voix.

Léon Gontran Damas (1912-1978) est, selon toute vraisemblance, un poète en blès. Sa vie est marquée par les drames et son enfance guyanaise stigmatisée par la mort : celle de sa sœur jumelle, une blessure infligée dans sa première année, dont il ne se remettra pas, mais dont il ne parle jamais. Puis viendra le décès de sa mère, alors qu'il n'a qu'un an, et celui de sa grand-mère, dont la mise en bière le traumatise au point qu'il devient muet pendant cinq ans. Quand il recouvre la parole, en 1919, le petit Damas commence ses études primaires. Il se retrouve ensuite, en 1924, au lycée Victor-Schœlcher de Fortde-France, en Martinique. Il y deviendra l'ami d'Aimé Césaire, camarade de lutte avec lequel il fondera plus tard le mouvement de la Négritude, mais qui sera surtout son confident.

19 Léon Gontran Damas est engagé et de cela, il ne se cache pas. Ses poèmes, rassemblés dans la multitude de recueils qu'il publie de son vivant, et notamment Pigments et Black Label qui paraît en 1956, sont des pamphlets brûlants. La censure coloniale ne lui fait pas peur et il en dénonce l'iniquité; il ravive cette partie de l'histoire française qui 
s'achève à peine: la colonisation, et dont on ne veut déjà plus entendre parler. L'écriture de Damas, sa verve poétique, est éminemment politique; il fait siennes les grandes causes anticoloniales, la résistance et la dénonciation. Le poème intitulé La complainte du nègre est exemplaire du verbe lourd, rude et direct qui caractérise Damas:

Ils me l'ont rendue
la vie
plus lourde et lasse

Mes aujourd'hui ont chacun sur mon jadis

de gros yeux qui roulent de rancœur

de honte

Les jours inexorablement

tristes

jamais n'ont cessé d'être

à la mémoire

de ce que fut

ma vie tronquée

Va encore

mon hébétude

du temps jadis

de coups de corde noueux

de corps calcinés

de l'orteil au dos calcinés

de chair morte

de tisons

de fer rouge

de bras brisés

sous le fouet qui se déchaîne

sous le fouet qui fait marcher la plantation

et s'abreuver de sang de mon sang de sang la sucrerie

et la bouffarde du commandeur crâner au ciel. ${ }^{12}$

Il y dénonce les exactions de l'esclavage, la torture, l'innommable. Cependant, si l'engagement public de Damas est réel et intense, de sa vie intime, de ses douleurs, de ses émois et de ses espérances, il ne dit rien. Rien des traumatismes qui ont marqué son enfance, rien de ses attirances sexuelles, rien. Il s'autocensure, effaçant sa douleur, enfermant sa blès dans une « kumbla » d'oubli et de déni qui suinte parfois à peine ici et là. ${ }^{13}$

21 Dans une stratégie de révélation, de réparation et de dé-censure, Roland Brival vient en quelque sorte, dans Nègre de personne, achever l'œuvre de Léon Gontran Damas. C'est par un roman que l'écrivain martiniquais apporte une voix libérée au poète guyanais, une voix qui s'adresse à un autre poète, le comparse de toujours, « le point d'ancrage de la triade » tel que le nomme le Damas brivalien, Aimé Césaire. ${ }^{14}$ Tout le roman est une longue confidence, un long récit, un long aveu à Césaire, comme le poète martiniquais est nommé dans le roman. Le ton est épistolaire mais le roman ne contient pas une seule lettre. Dans Nègre de personne, Césaire est un compagnon inséparable, celui qui est loin, à Paris ou à Fort-de-France, celui, qui a su crier sa "blessure sacrée ", et qui accompagne Damas dans sa longue remontée des souvenirs traumatiques, dans son exploration de la passion, dans la révélation des dérives. Sous la plume de Brival, Damas s'épanche, se confie, ce qu'il n'a jamais pu faire, de son encre et de son vivant. 
Libéré par Brival des interdits coloniaux, du poids des jugements, de la culpabilité et de la peur, Damas convoque Césaire à l'écoute et au partage pour qu'il devienne en quelque sorte le témoin et l'aidant indispensable à cette longue dé-censure :

Les rues de Paris, sans toi, ont perdu leur charme à mes yeux. Les cafés où nous allions nous attabler pour d'interminables discussions, les bibliothèques que nous fréquentions, les couloirs de la Sorbonne où nous errions les bras chargés de livres, les allées du jardin du Luxembourg [...] tout cela n'est plus qu'un paysage déserté, dont seul je n'ai plus la force de recomposer l'essentiel. [...]

Un matin à mon réveil j'ai su qu'il était temps de m'arracher à l'humeur morbide qui m'emprisonnait. C'est alors que m'est venue cette idée dont l'évidence m'a aussitôt terrassé. Partir en Amérique. ${ }^{15}$

Le roman commence donc avec l'arrivée à New-York, et sera le récit d'une longue errance dans la ville, mais aussi et surtout un long cheminement des pieds à la tête comme le dirait le poète et peintre trinidadien Leroy Clarke, une plongée en soi. Le Damas brivalien donne le ton « quoique j'y vienne en guerrier... c'est l'émotion trouble d'un adolescent qui perdure en moi à l'instant de descendre les marches de cette passerelle ${ }^{16}{ }^{16}$ Ainsi et enfin dé-censuré, sorti de ces interdits qu'il s'inflige à lui-même, Damas révèle, dans Nègre de personne, ses grandes douleurs : la mort, le viol... et le silence, refuge obligé pour survivre, comme l'esclave à la peau déchirée par le fouet :

Jusqu'à l'âge de six ans, Césaire, je n'ai prononcé aucun mot. Les gens me croyaient muet. J'étais coincé dans la case du silence comme un gosse effrayé par la rumeur $\mathrm{du}$ jour. Et je ne te parle pas du silence colonial où des siècles d'indigence ont voulu nous plonger, mais d'un silence plus vaste encore, et dont il faut parfois une vie entière pour dénouer le mystère.

Je le dois à ma sœur jumelle, Gabrielle. Imagine-nous blottis l'un contre l'autre dans le refuge du ventre maternel, et tu sauras de quelle appartenance nous partagions le fruit. Son souffle était le mien. Mes pensées étaient les siennes. Nous vivions soudés à la racine d'un même corps. Pourtant, elle est morte quelques jours après notre naissance, comme si elle n'avait eu l'intention que de m'accompagner jusqu'à la porte du monde, avant de repartir danser sous d'autres soleils.

Rien ne saurait se comparer à la stupeur inouïe que provoque un tel naufrage. Voilà comment, sans doute, s'est installée cette douleur corrosive qui vit désormais accrochée à la plume de mon stylo. Un an plus tard j'ai perdu ma mère. Puis ce fut au tour de ma grand-mère...

J'étais l'éponge de toutes les larmes enfouies. L'aimant de toutes les frustrations... ${ }^{17}$

Enfin, le pus s'écoule, sous la plume tendre et révélatrice de Brival, le guerrier s'effondre, fait face à sa blès, révèle la vérité essentielle. C'est une reconstruction qui s'opère dans l'écriture de Brival, une réparation de l'être. À partir de la reconnaissance de ce premier trauma, l'écrivain martiniquais procède comme à un épanchement puissant, non pas du militant, mais de l'être sensible qu'est Damas. Poète à la place du poète, Brival oscille dès lors de révélations libératrices en dérives poétiques, rêvant pour Damas de « pluie de [...] mots », «d'atteindre l'extase en composant [des] vers ».18 Brival explore tous les silences, tous les maux censurés : le viol perpétré contre le petit Léon par un prêtre dépravé que le Damas brivalien commence à évoquer avec ces mots :

Je ne t'ai jamais parlé, Césaire, de ce drame intime, trop personnel pour être livré même à mes amis. Du moins l'ai-je cru. Mais ce soir, peut-être à cause de la distance qui nous sépare, me voilà en train de remuer toutes ces choses dans ma mémoire... Cela commence par un après-midi du mois de juillet, avec un ciel limpide... mais ce jour là, arrivé à la porte du presbytère je trouve l'endroit désert... La séance a été annulée me dit le prêtre... mais je t'enseignerai quand même ce qu'il faut savoir... M'attrapant par les épaules, il plonge ses yeux dans les miens, puis le voilà qui me susurre un discours inédit sur la volonté divine à laquelle tous les élus du Christ - 
dont je suis précise-t-il - ont le devoir d'obéir... La soutane part à la volée sur une chaise...il me soulève et me renverse sur le divan étroit... Je suffoque... Le reste de la scène s'est effacé à jamais de ma mémoire, dispersé aux quatre vents comme le furent ce jour-là, les cendres de mon enfance. ${ }^{19}$ C'est pour échapper à la dépravation violente du prêtre mais aussi au scandale, que la Tante Gabi envoie le jeune Damas en Martinique, où il devient pensionnaire au lycée Schœlcher ; c'est là, sur les bancs de l'école, qu'il va rencontrer Césaire, son ami, son confident. Ce ne sont, heureusement, pas seulement des révélations aussi tragiques que Brival dé-censure : nulle intention pour l'écrivain de poser Damas en victime et de larmoyer à sa place : «Vois-tu [...] je n'ai pas l'âme d'une victime. Mes plaies et mes erreurs sont toujours à porter au bénéfice du compte, plutôt qu'à son débit » :20

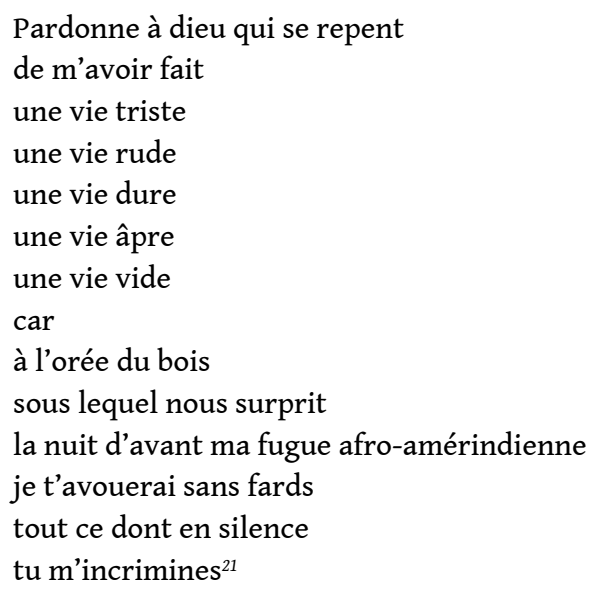

Ainsi, Brival va également explorer et raconter toute une expérience, parfois joyeuse, parfois surprenante, de passions pour deux femmes : une jeune peintre haïtienne, Anna, qui lui fait découvrir les mystères du vaudou, et passion pour la chanteuse de jazz Billie Holliday, à qui il voue un véritable culte, et qui l'entraînera dans les dérives et les délires de l'alcool et de la drogue sur fond de jazz. Du militantisme avec la NAACP, ${ }^{22}$ à toutes les émotions puissantes qu'il vit avec Anna et Billie, Damas, sous la plume de Brival, raconte tout : il parle enfin, non plus en homme de l'espace public mais à partir de l'intimité de son cœur.

L'intimité est aussi l'espace qu'Olive Senior explore et sonde dans son roman, qui est structuré comme un recueil de nouvelles; en effet The Pain Tree rassemble dix récits. Cependant, l'ouvrage constitue bel et bien un ensemble romanesque cohérent puisqu'il existe un lien entre les personnages. Ce lien qui est rhizomique plonge ses racines dans plusieurs cadres et expériences de vie qui tournent tous autour de cette femme étouffante et stérilisante qui n'est autre que la mère de l'héroïne qui apparait comme une représentation autobiographique de Senior, tantôt une enfant, tantôt une jeune femme, témoin de tous ces destins mêlés, la voix qui va donner voix à tous les protagonistes. C'est à travers elle qu'Olive Senior se livre à une véritable exploration cathartique de la société coloniale jamaïcaine, de l'imposition culturelle coloniale que symbolise la mère, véritable tyran qui impose à tous l'ordre, le silence et la peur. C'est également ce personnage transversal qui révèlera le sens et la fonction de l'arbre à douleur, déversoir et mémoire des souffrances du peuple jamaïcain.

Aussi, Olive Senior, dans The Pain Tree, s'applique-t-elle aussi à nettoyer et dépasser la blès en faisant exploser les cadres rigides et restrictifs des oppositions binaires qui ont

Transtext(e)s Transcultures 跨文本跨文化, 15 | 2020 
toujours défini le système plantationnaire. ${ }^{23}$ Vérité-mensonge, blanc-noir, pauvreriche, éduqué-ignorant, ces oppositions se fissurent et tout se mêle pour révéler dans un autre langage, un langage repensé, restructuré, nuancé, un espace de parole qui ne méprise aucun être, aucune des modalités d'expression, aucun sentiment.

Ainsi, étrangement, vont apparaître, dans The Pain Tree, les mêmes thématiques liées à la censure de la langue et de l'affect que dans Nègre de personne: un petit garçon muet pendant des années qui ici s'appelle tout simplement Boy, un ecclésiastique violeur qui impose le silence, une jeune fille en quête d'esthétisme. Mais ce roman-recueil s'articule aussi autour d'une parole de femme libérée, la parole d'olive Senior ellemême, dont la conscience poétique et politique, fondamentalement jamaïcaine et afrodescendante, avait été censurée par sa mère blanche.

Senior, en choisissant la posture de l'observateur-observé, de l'écrivain qui possède les clés du système de représentation qui est le sien, propose au lecteur une écriture et un récit débarrassés de l'ethnocentrisme que symbolise sa mère et qui censure "les fondamentaux de la nature humaine: le magique, le syncrétique, l'intuitif et le sensoriel. Ici, les barrières entre deux mondes différents, [...] celui de la pensée structurée et l'autre, celui de la pratique magique, de la création et de l'intuitif sont abolies. $»^{24}$ Ainsi Senior, à travers ce long monologue dans lequel une multitude de personnages s'expriment, se positionne en voix caribéenne, elle entre en résistance, non seulement contre cette censure coloniale qui interdit l'émission de pensées et de paroles différentes et échappant à la compréhension d'une élite coloniale monolithique ; mais la romancière jamaïcaine fait également le choix d'une écriture qui s'inscrit dans une esthétique caribéenne. En effet, l'écriture de Senior s'inscrit dans la restitution des pratiques, des représentations et des modalités de la pensée et des discours caribéens. Sa manière de camper ses personnages ruraux, de mettre en scène leur gestuelle et leurs habitus prend ancrage dans «le génie populaire» et l'arbre: «the pain tree » est "l'expression des croyances, la représentation des rituels et la définition même de l'espace et de l'artefact comme lieu et objet spirituel... ». ${ }^{25}$

À la fin du cheminement de la parleuse qui va maison après maison, vie après vie, prêter sa voix à chacun de ceux qui étaient restés muets, la dé-censure s'applique. Finalement, non seulement la voix comprend le fonctionnement de l'arbre qu'est « The Pain Tree ", mais elle réussit aussi à l'utiliser. En effet, cet arbre est le réceptacle, le gardien des paroles des âmes endolories, des vies en blès. Et lorsque la vie et la douleur deviennent insupportables, on plante un clou dans l'arbre : le Fromager. La voix, qui n'avait jamais réussi à le faire, enfonce enfin son propre clou et parvient à dé-censurer sa propre parole, celle d'une petite fille empêchée par la voix écrasante de sa mère de dire à son pays et à elle-même ses paroles d'amour.

\section{Conclusion}

31 Ce clou que les personnages d'olive Senior viennent tour à tour planter dans le fromager, l'arbre sacré, permet de guérir la douleur en la transmettant au géant épineux. La dé-censure que pratiquent Roland Brival et Olive Senior dans leurs romans contemporains, est un moment important non seulement de la littérature caribéenne, mais aussi de l'histoire caribéenne; car ces deux auteurs nous apportent, par leur écriture, une clé, une compréhension du sujet caribéen. Les stratégies de dé-censure qu'ils appliquent dans leurs récits (repenser le langage, décloisonner le verbe, 
décentrer la pensée, reconstruire la parole) sont des stratégies édificatrices qui permettent une réparation de l'être et de l'âme et une guérison de la blès.

C'est aussi par la résistance à la censure, par cette dé-censure du verbe que les deux auteurs épousent véritablement l'esthétique caribéenne en introduisant la mémoire, la spiritualité et le génie populaire comme piliers fondateurs de leur écriture ; car comme je l'explique dans :

[...] dans tous les domaines artistiques, les créateurs n'ont eu de cesse d'essayer de développer un vocabulaire, une écriture, des thématiques, des formes, des combinaisons, des arrangements distincts et propres non seulement à les définir en tant que caribéens, mais surtout propres à permettre l'expression de ressentis variés, difficiles parfois et souvent confus, venant de l'inadéquation patente entre des systèmes de représentation eurocentrés et la réalité caribéenne des artistes. ${ }^{26}$

Roland Brival et Olive Senior choisissent, par le choix de la dé-censure qu'ils pratiquent dans leur œuvre respective, d'inscrire leur œuvre dans un enracinement insurgé. Dans un renouvellement des stratégies d'écriture qui échappent à la fois à toute déviance exotique, au monolithisme passéiste et au poids des interdits, ils entrent véritablement en adéquation avec leur réalité.

\section{NOTES}

1. Odile Goerg, « Entre infantilisation et répression coloniale Censure cinématographique en AOF, "grands enfants" et protection de la jeunesse ", in Cahier d'études africaines, 205, 2012, Varia, p. 165-198.

2. Roland Brival, Nègre de personne, Paris, Gallimard, 2016.

3. Aimé Césaire, Discours sur le colonialisme, Paris, Présence Africaine, 1955 (2004), p. 91.

4. Frantz Fanon, Les Damnés de la terre, Paris, François Maspéro, (La Découverte 2011), 1961, p. 27.

5. Frantz Fanon, Peau noire, Masques blancs, Paris, Éditions du Seuil, 1952, p. 216, 217.

6. Aimé Césaire, Cahier d'un retour au pays natal, Paris, Présence africaine, (1983) 1947, p. 22.

7. Négritude dont Léon Gontran Damas, qui a connu Aimé Césaire dans son enfance au Lycée Schœlcher, est membre fondateur.

Césaire, Discours, p. 23.

8. Carole Boyce Davies, Elaine Savory Fido, Out of the Kumbla: Caribbean Women and Literature, Trenton, Africa World Press, 1990.

9. Patricia Donatien-Yssa, L'exorcisme de la blès - Vaincre la souffrance dans Autobiographie de ma mère de Jamaica Kincaid, Paris, Le Manuscrit, 2006, p. 16.

10. Daniel Maximin, «Léon Damas, étoile de graffiti » in Emina, Antonella (dir.), Léon-Gontran Damas. Cent ans en noir et blanc, Paris, CNRS Éditions, 2014.

11. Maximin, « Léon Damas, étoile de graffiti », p. 15.

12. Léon Gontran Damas, Pigments, Paris, Guy Lévi Mano, 1937.

13. Calebasse.

14. Brival, Nègre de personne, p. 12. Le Damas qui dans l'autobiographie fictive s'exprime par la voix de Brival.

15. Brival, Nègre de personne, p. 13.

16. Brival, Nègre de personne, p. 17. 
17. Brival, Nègre de personne, p. 28.

18. Brival, Nègre de personne, pp. $30,88$.

19. Brival, Nègre de personne, pp. 65-67.

20. Brival, Nègre de personne, p. 68.

21. Léon Gontran Damas, Graffiti, Paris, Éditions Seghers, 1952 (extrait).

22. National Association for the Advancement of Colored People.

23. Olive Senior, The Pain Tree, Toronto, Cormorant Books, 2015.

24. Patricia Donatien, L'art caribéen, le penser pour le dire. Réflexions autour de la littérature, des arts visuels, de la musique et de la danse, Paris, L'Harmattan, 2018, pp. 56, 57.

25. Donatien, L'art caribéen, le penser pour le dire, p. 99.

26. Donatien, L'art caribéen, le penser pour le dire, p. 78.

\section{RÉSUMÉS}

Roland Brival et Olive Senior sont tous les deux des auteurs caribéens contemporains issus de sociétés coloniales hautement dirigistes et conformistes et cependant, ils pratiquent la décensure. Leurs œuvres sont en effet des outils et des espaces dans lesquels l'indicible et l'inaudible vont pouvoir être exprimés, au travers d'un certain nombre de stratégies d'écriture clairement identifiables comme relevant d'une esthétique caribéenne. Les deux romans présentés dans cet article sont tous deux récents ; il s'agit, d'une part, de Nègre de personne que Brival publie en 2016 et qui est très singulier en ce sens qu'il met en scène une partie de la vie du poète guyanais Léon Gontran Damas sous la forme d'un récit autobiographique. Dans ce roman le poète raconte son voyage à New York en 1939, et, c'est en ce sens qu'il est singulier, Brival y substitue son écriture et sa voix à celles de Damas. Le deuxième roman publié en 2015 par Olive Senior est une évocation de plusieurs récits de vie, tous mis en relation par deux axes récurrents et incontournables dans chaque évocation, une femme, la mère de l'auteur, et un arbre dont le roman porte d'ailleurs le nom The Pain Tree. Dans ces deux œuvres, la censure qui touche aussi bien l'affect que la langue et l'écriture, n'est pas celle que subissent leurs auteurs; elle est celle qu'ont subie d'une part Léon Gontran Damas et d'autre part les personnages d'olive Sénior. Tous sont victimes d'un empêchement, d'une impossibilité, d'une censure de leur parole et de leur écriture due aux restrictions des droits des sujets coloniaux, à la rigidité d'une institution et de sociétés marquées par le non-humanisme esclavagiste et qui impose «l'amnésie comme méthode » (Césaire Discours sur le colonialisme, p.91). L'article est également fondé sur le concept d'imposition culturelle avancé par le médecin-psychiatre et écrivain Frantz Fanon qui démontre dans son ouvrage Les Damnés de la terre comment le monde colonial repose sur la violence, y compris culturelle. Ainsi, cette étude a aussi pour ambition d'étudier la censure comme forme de violence coloniale qui prévient autant qu'elle réprime toute production qui n'obéit pas à cette imposition culturelle. L'objet principal de ce travail est donc de démontrer comment les œuvres de Roland Brival et Olive Senior participe d'une dé-censure des voix et des œuvres littéraires caribéennes de la première moitié du $\mathrm{XX}^{\mathrm{e}}$ siècle.

Roland Brival and Olive Senior are both contemporary Caribbean authors from highly interventionist and conformist colonial societies and yet they practice de-censorship. Their works are indeed tools and spaces in which the unspeakable and the inaudible are expressed through a number of writing strategies clearly identifiable as belonging to Caribbean aesthetic. 
The two novels presented in this article are both recent; it is on the one hand, Nègre de personne published by Roland Brival in 2016 and which is very singular in the sense that it stages part of the life of the Guyanese poet Léon Gontran Damas in the form of an autobiographical story. In this novel, the poet recounts his trip to New York in 1939 and, rather surprisingly, Brival, all along the text, substitutes his writing and his voice to Damas'ones. The second novel published in 2015 by Olive Senior is an evocation of several life stories, all linked by two recurring and essential axes in each evocation, a woman, the author's mother and a tree whose name is also the title of the novel The Pain Tree. In these two works, the censorship, which touches affect as well as language and writing, is not that suffered by their authors; it is the one suffered by Léon Gontran Damas on the one hand and Olive Senior's characters on the other hand. All are victims of an impediment, an impossibility, a censorship of their speech and their writing due to the restrictions on the rights of colonial subjects, the rigidity of an institution and of societies marked by slavery non-humanism and which imposes "amnesia as a method" (Césaire, Discours sur le colonialisme, p.91). The article is also based on the concept of cultural imposition advanced by the physician-psychiatrist and writer Frantz Fanon who demonstrates in his book Les Damnés de la Terre how the colonial world is based on violence, including cultural violence; thus, this study also aims to study censorship as a form of colonial violence that prevents as much as it represses any production that does not obey this cultural imposition. The main object of this writing is to demonstrate how the works of Roland Brival and Olive Senior participate in a decensorship of Caribbean voices and literary works from the first half of the 20th century.

\section{AUTEUR}

\section{PATRICIA DONATIEN}

Patricia Donatien est Professeur des Universités à l'Université des Antilles, son travail de recherches qui complète ses activités d'artistes l'a amenée à écrire une trentaine d'articles sur les arts visuels et la littérature et deux ouvrages L'exorcisme de la blès primé en 2008 par le Frantz Fanon Prize de la Caribbean Philosophical Association et L'art Caribéen le penser pour le dire 2018. Elle exerce aussi en tant que commissaire d'exposition. Également danseuse et musicienne, Patricia Donatien œuvre dans le militantisme culturel, son travail plastique engagé aborde les questions de la mémoire, de la réparation, de la transmission et de la dénonciation des injustices qui impactent encore les sociétés caribéennes.

Patricia Donatien is a University Professor at the University of the West Indies, her research work which complements her artistic activities has led her to write about thirty articles on visual arts and literature and two books L'exorcisme de la blès awarded in 2008 by the Frantz Fanon Prize of the Caribbean Philosophical Association and L'art Caribéen le penser pour le dire 2018. She also works as an exhibition curator. Also dancer and musician, Patricia Donatien works in cultural activism, her engaged plastic work addresses the issues of memory, reparation, transmission and denunciation of the injustices that still impact Caribbean societies. 